Notre Dame Journal of Formal Logic

Volume 40, Number 4, Fall 1999

\title{
Completeness and Definability in the Logic of Noncontingency
}

\author{
EVGENI E. ZOLIN
}

\begin{abstract}
Hilbert-style axiomatic systems are presented for versions of the modal logics $\mathbf{K} \Sigma$, where $\Sigma \subseteq\{\mathbf{D}, \mathbf{4}, \mathbf{5}\}$, with noncontingency as the sole modal primitive. The classes of frames characterized by the axioms of these systems are shown to be first-order definable, though not equal to the classes of serial, transitive, or euclidean frames. The canonical frame of the noncontingency logic of any logic containing the seriality axiom is proved to be nonserial. It is also shown that any class of frames definable in the noncontingency language contains the class of functional frames, and dually, there exists a greatest consistent normal noncontingency logic.
\end{abstract}

1 Introduction The noncontingency operator $\Delta$ is defined in terms of the necessity operator $\square$ by putting $\Delta A:=\square A \vee \square \neg A$. This induces a translation of $\Delta$-formulas (i.e., formulas in the propositional modal language with $\Delta$ as the sole modal primitive, $\Delta$-language for short) into $\square$-formulas. So, to any $\square$-logic $L$ (i.e., logic in the $\square$ language) one can associate a noncontingency logic of $L$, denoted by $L^{\Delta}$, consisting of all $\Delta$-formulas whose translations are theorems of $L$.

Montgomery and Routley $[8]$ axiomatized the noncontingency logics of T, S4, and $\mathbf{S 5}$ (see also 91 and [10]). It is worth noting that in case when $L$ contains $\mathbf{T}$, or more specifically, the reflexivity scheme $\square A \rightarrow A$, necessity is definable in terms of noncontingency ( $\Delta$-definable, for short) by $\square A=A \& \Delta A$. In the logic Ver, the same effect is observed: it proves, for any $A$, a formula $\square A \leftrightarrow \top$, which can be regarded as a $\Delta$-definition of $\square$. Cresswell [3] provides an example of logic $\mathbf{H}$ such that $\mathbf{H} \nsupseteq \mathbf{T}, \mathbf{H} \neq \mathbf{V e r}$, but $\square$ is $\Delta$-definable in $\mathbf{H}$.

A systematic study of noncontingency logic, in particular, the cases when $\square$ is not $\Delta$-definable, was initiated by Humberstone. In his paper [4], a (rather complicated) system axiomatizing the noncontingency logic of K was presented. Kuhn [5] succeeded in simplifying this system and proposed a finite axiomatization of the noncontingency logic of $\mathbf{K} \mathbf{4}$.

Received October 10, 2000; revised February 12, 2001 
Our paper continues this line of investigation. In Section 2 we present finite axiomatization for the $\operatorname{logics} \mathbf{K} \Sigma^{\Delta}$, where $\Sigma \subseteq\{\mathbf{4}, \mathbf{5}\}$. We have slightly modified the axiomatization of $\mathbf{K}^{\Delta}$ and $\mathbf{K 4}^{\Delta}$ suggested by Kuhn 5 to make it similar to the standard axiomatization of normal logics. In Section 3 we discuss a problem with canonical model construction for the logic $\mathbf{D}^{\Delta}$; it appears that if a normal logic $L$ contains the seriality axiom then the canonical frame of $L^{\Delta}$ is not serial and hence falsifies $L$. However, there is a detour way to find an axiomatization of some of these noncontingency logics; namely, adding the seriality axiom to some logics does not change the noncontingency logic thereof. Section 4 s concerned with definability of classes of frames by sets of $\Delta$-formulas. Here we show that the class of functional frames is the smallest $\Delta$-definable class; in particular, this explains why most of $\square$-definable classes of frames are not $\Delta$-definable. Dually, in Section 3 the logic $\operatorname{Ver}^{\Delta}$ is shown to be the greatest in the lattice of consistent normal noncontingency logics. In the final Section 5 first-order formulas are found that correspond to (i.e., characterize the same classes of frames as) the axioms of noncontingency logics mentioned in Section 2.

The logic KD45 is known to capture the principles of reasoning involving epistemic judgments: the postulates of this logic are valid under the (informal) interpretation of a sentence of the form $\square A$ as " $A$ is known (to some idealized person)". In this context, the noncontingency assertion $\Delta A$ means "the truth value of $A$ is known to the person". (Strictly speaking, the logic KD45 is usually proposed to represent categorical beliefs, when $\square A$ is read " $A$ is believed (by an idealized person)", for this logic does not contain the axiom $\square A \rightarrow A$ and hence allows the person to believe in false statements. In accordance with this, doxastic, understanding of the modality ' $\square$ ', the assertion $\Delta A$ means "the person has a definite view-point concerning the truth value of $A$ ".) In Section 3 the logic of this modality, that is, the noncontingency logic of KD45, is shown to coincide with that of K45.

2 Transitive euclidean noncontingency logic The propositional modal language consists of a denumerable set of variables $\operatorname{Var}=\left\{p_{0}, p_{1}, \ldots\right\}$, symbols for falsehood $\perp$, implication $\rightarrow$, and a unary modal operator $\square$. Other connectives $(\top, \neg, \&, \vee, \leftrightarrow$, $\diamond)$ are taken as standard abbreviations. The set of formulas of this language is defined as usual and is denoted by $\mathbf{F m} \square$. This language will be referred to as a $\square$-language and its formulas as $\square$-formulas. A $\Delta$-language and the set $\mathbf{F m}^{\Delta}$ of $\Delta$-formulas are defined similarly. We fix a natural translation $\operatorname{tr}: \mathbf{F m}^{\Delta} \rightarrow \mathbf{F} \mathbf{m}^{\square}$ which respects Boolean connectives and $\operatorname{tr}(\Delta A):=\square \operatorname{tr}(A) \vee \square \neg \operatorname{tr}(A)$.

A (Kripke) frame is a structure $\langle W, \uparrow\rangle$, where $W$ is a nonempty set of "worlds" and $\uparrow$ is a binary "accessibility" relation on $W$. By $\downarrow$ we denote the converse relation of $\uparrow$. A model $M=\langle F, \models\rangle$ consists of a frame $F$ and a valuation $\models \subseteq W \times$ Var. The notion " $A$ is true in $M$ at $w$ " (written $M, w \models A$ and $M$ usually omitted) is defined for both $\square$ - and $\Delta$-formulas in the standard way; the modal clauses are as follows:

$$
\begin{aligned}
& w \models \square A \quad \leftrightharpoons \quad \forall x \downarrow w \quad x \models A ; \\
& w \models \Delta A \quad \leftrightharpoons \quad(\forall x \downarrow w \quad x \models A) \text { or }(\forall x \downarrow w \quad x \not \equiv A) .
\end{aligned}
$$

Obviously, $w \models A \Leftrightarrow w \models \operatorname{tr}(A)$, for any $\Delta$-formula $A$. A formula $A$ is valid in a frame $F(F \models A$, in symbols) if $A$ is true at every world in every model based on $F$. 
If $\Gamma$ is a formula or a set of formulas then a $\Gamma$-frame is a frame validating $\Gamma$; the class of all $\Gamma$-frames is denoted by $\mathcal{F}(\Gamma)$.

A $\square$-logic is a set of $\square$-formulas containing all classical tautologies and closed under the rules of modus ponens, substitution, and equivalent replacement:

$$
\text { (MP) } \left.\frac{A \quad A \rightarrow B}{B} \quad \text { (Sub) } \frac{A}{A[B / p]} \quad \text { (RE }^{\square}\right) \frac{A \leftrightarrow B}{\square A \leftrightarrow \square B} .
$$

(Here $A[B / p]$ is the result of substituting a formula $B$ for all occurrences of a variable $p$ in $A$.) The notion of $\Delta$-logic is defined similarly. Given a $\square$-logic $L$, a noncontingency logic of $L$ (briefly, a $\Delta$-logic of $L$ ), denoted by $L^{\Delta}$, is the set of all $\Delta$-formulas whose translations are theorems of $L$ (it is indeed a $\Delta$-logic):

$$
L^{\Delta}=\left\{A \in \mathbf{F m}^{\Delta} \mid \operatorname{tr}(A) \in L\right\}=\operatorname{tr}^{-1}(L) .
$$

The minimal normal modal logic $\mathbf{K}$ has the rules (MP), (Sub), and the following axioms and the "necessitation" rule:

$$
\begin{array}{l|l}
\text { ( } \mathrm{A}_{T}^{\square} \text { ) All classical tautologies in the } \square \text {-language } & \text { (Nec) } \frac{A}{\square A} \\
\text { ( } \left.\mathrm{A}_{\mathbf{K}}^{\square}\right) \square(p \rightarrow q) \rightarrow(\square p \rightarrow \square q) \quad \text { (distributivity) } &
\end{array}
$$

In this paper we consider the systems $\mathbf{K} \Sigma, \Sigma \subseteq\{\mathbf{D}, \mathbf{4}, \mathbf{5}\}$, obtained by adding to $\mathbf{K}$ the axioms $\left(A_{\mathfrak{S}}^{\square}\right), \mathfrak{S} \in \Sigma$, listed below (the class of frames characterized by $\left(A_{\mathfrak{S}}^{\square}\right)$ is first-order definable by a formula $\left(\varphi_{\mathfrak{S}}^{\square}\right)$ also shown below).

$$
\begin{array}{l|lll}
\left(\mathrm{A}_{\mathbf{D}}^{\square}\right) \square p \rightarrow \diamond p & \left(\varphi_{\mathbf{D}}^{\square}\right) \forall w \exists x \quad w \uparrow x & & \text { (seriality) } \\
\left(\mathrm{A}_{\mathbf{4}}^{\square}\right) \square p \rightarrow \square \square p & \left(\varphi_{\mathbf{4}}^{\square}\right) \forall w \forall x \downarrow w \forall y \downarrow x & w \uparrow y & \text { (transitivity) } \\
\left(\mathrm{A}_{\mathbf{5}}^{\square}\right) \nabla p \rightarrow \square \diamond p & \left(\varphi_{\mathbf{5}}^{\square}\right) \forall w \forall x \downarrow w \forall y \downarrow w & x \uparrow y & \text { (euclideanness) }
\end{array}
$$

Now we formulate our axiomatic systems for $\Delta$-logics of $\mathbf{K} \Sigma, \Sigma \subseteq\{\mathbf{4}, \mathbf{5}\}$; logics containing the seriality axiom are considered in the next section. For notation simplicity, we denote the systems by $\mathbf{K} \Sigma^{\Delta}$; Theorem 2.2 below justifies the notation. The logic $\mathbf{K}^{\Delta}$ has the rules (MP) and (Sub) as well as the following axioms and the "noncontingentization" rule (cf. [4]):

$$
\begin{array}{ll|l}
\left(\mathrm{A}_{\mathrm{T}}^{\Delta}\right) \text { All classical tautologies in the } \Delta \text {-language } & \\
\left(\mathrm{A}_{\mathbf{K}}^{\Delta}\right) \Delta(p \leftrightarrow q) \rightarrow(\Delta p \leftrightarrow \Delta q) & \text { (equivalence) } & \text { (mCR) } \frac{A}{\Delta A} \\
\left(\mathrm{~A}_{\triangle}^{\Delta}\right) \Delta p \leftrightarrow \Delta \neg p & \text { (mirror axiom) } & \\
\left(\mathrm{A}_{\vee}^{\Delta}\right) \Delta p \rightarrow[\Delta(q \rightarrow p) \vee \Delta(p \rightarrow r)] & \text { (dichotomy) } &
\end{array}
$$

To obtain the system $\mathbf{K} \Sigma^{\Delta}, \Sigma \subseteq\{\mathbf{4}, \mathbf{5}\}$, add to $\mathbf{K}^{\Delta}$ the relevant axioms:

$$
\begin{array}{ll}
\left(\mathrm{A}_{4}^{\Delta}\right) \Delta p \rightarrow \Delta(q \rightarrow \Delta p) & \text { (weak transitivity). } \\
\left(\mathrm{A}_{5}^{\Delta}\right) \neg \Delta p \rightarrow \Delta(q \rightarrow \neg \Delta p) & \text { (weak euclideanness). }
\end{array}
$$

Clearly, these systems are closed under the rule $\left(\mathrm{RE}^{\Delta}\right)$.

Before we pass to the main result of this section, let us recall for the future reference the axiomatization of $\Delta$-logics of $\mathbf{T}, \mathbf{S 4}$, and S5 (cf. 80 and [9). First, T, S4, and $\mathbf{S 5}$ are axiomatized over $\mathbf{K}$ as follows:

$$
\mathbf{T}=\mathbf{K}+\left(A_{\mathbf{T}}^{\square}\right), \quad \mathbf{S 4}=\mathbf{T}+\left(A_{\mathbf{4}}^{\square}\right), \quad \mathbf{S 5}=\mathbf{T}+\left(A_{\mathbf{5}}^{\square}\right),
$$


where $\left(\mathrm{A}_{\mathbf{T}}^{\square}\right.$ ) is the reflexivity axiom $\square p \rightarrow p$. The $\Delta$-logics of $\mathbf{T}, \mathbf{S 4}$, and $\mathbf{S 5}$ can be axiomatized over $\mathbf{K}^{\Delta}$, however, in 8 and 9 the following simple axiomatization thereof is proposed. The rules of $\mathbf{T}^{\Delta}$ are (MP), (Sub), and (NCR) and the axioms are $\left(\mathrm{A}_{T}^{\Delta}\right),\left(\mathrm{A}_{\neg}^{\Delta}\right)$, and

$$
\left(\mathrm{A}_{\mathbf{T}}^{\Delta}\right) p \rightarrow[\Delta(p \rightarrow q) \rightarrow(\Delta p \rightarrow \Delta q)] \quad \text { (weak distributivity) }
$$

The logics $\mathbf{S 4}^{\Delta}$ and $\mathbf{S 5}^{\Delta}$ are axiomatized as follows:

$$
\mathbf{S 4}^{\Delta}=\mathbf{T}^{\Delta}+\left(\mathrm{A}_{\mathbf{4} b}^{\Delta}\right), \quad \mathbf{S 5}^{\Delta}=\mathbf{T}^{\Delta}+\left(\mathrm{A}_{\mathbf{5} b}^{\Delta}\right)=\mathbf{T}^{\Delta}+\left(\mathrm{A}_{\mathbf{5}^{\prime}}^{\Delta}\right),
$$

where the extra axioms are

$$
\begin{array}{lll}
\left(\mathrm{A}_{4 b}^{\Delta}\right) & \Delta p \rightarrow \Delta \Delta p & \text { (b-transitivity) } \\
\left(\mathrm{A}_{\mathbf{5 b}_{b}}^{\Delta}\right) & \Delta \Delta p & \text { (b-euclideanness) } \\
\left(\mathrm{A}_{\mathbf{5}^{\prime}}^{\Delta}\right) & \Delta(\Delta p \rightarrow p) & \text { (weak euclideanness). }
\end{array}
$$

The properties of frames expressed by the above-mentioned noncontingency axioms are presented in Section 5. There we show that classes of frames validating the axioms $\left(A_{4}^{\Delta}\right)$ and $\left(A_{4 b}^{\Delta}\right)\left(\right.$ respectively, $\left(A_{5}^{\Delta}\right),\left(A_{5 b}^{\Delta}\right)$, and $\left.\left(A_{5^{\prime}}^{\Delta}\right)\right)$ strictly contain the classes of transitive (respectively, euclidean) frames. The names we gave to these axioms can be partially justified by Theorem 5.3.

The main result of this section is formulated in the theorem below. It states that the systems $\mathbf{K} \Sigma^{\Delta}$ axiomatize exactly the $\Delta$-logics of $\mathbf{K} \Sigma, \Sigma \subseteq\{\mathbf{4}, \mathbf{5}\}$. For its proof, we use the canonical model argument adapted for $\Delta$-logics by Humberstone 44 and Kuhn [5]. For $\mathbf{K}^{\Delta}$ and $\mathbf{K} \mathbf{4}^{\Delta}$ the theorem is proved in [5], however, the axiomatization of these logics proposed in that paper differs slightly from ours, so we restate the result for our systems. We need an auxiliary lemma.

Lemma 2.1 $\mathbf{K}^{\Delta} \vdash \Delta p \& \Delta q \rightarrow \Delta(p \& q)$.

Proof: We derive in $\mathbf{K}^{\Delta}$ (derivations are written quite schematically):

$$
\mathbf{K}^{\Delta} \vdash \Delta[p \rightarrow q] \stackrel{1}{\leftrightarrow} \Delta[p \leftrightarrow(p \& q)] \stackrel{2}{\rightarrow}[\Delta p \rightarrow \Delta(p \& q)] .
$$

Here ' $\stackrel{1}{\leftrightarrow}$ ' is obtained from a tautology $[p \rightarrow q] \leftrightarrow[p \leftrightarrow(p \& q)]$ by applying the rule $\left(\mathrm{RE}^{\Delta}\right)$ and ' $\stackrel{2}{\rightarrow}$ ' is an instance of the axiom $\left(\mathrm{A}_{\mathbf{K}}^{\Delta}\right)$. Similarly,

$$
\mathbf{K}^{\Delta} \vdash \Delta[q \rightarrow p] \rightarrow[\Delta q \rightarrow \Delta(p \& q)] .
$$

Finally, we use the dichotomy axiom:

$$
\begin{aligned}
\mathbf{K}^{\Delta} \vdash \Delta p & \rightarrow\{\Delta(q \rightarrow p) \vee \Delta(p \rightarrow q)\} \rightarrow \\
& \rightarrow\{[\Delta p \rightarrow \Delta(p \& q)] \vee[\Delta q \rightarrow \Delta(p \& q)]\} \leftrightarrow \\
& \leftrightarrow\{(\Delta p \& \Delta q) \rightarrow \Delta(p \& q)\} .
\end{aligned}
$$

The first premise $\Delta p$ of $\Delta p \rightarrow\{(\Delta p \& \Delta q) \rightarrow \Delta(p \& q)\}$ is redundant.

Theorem 2.2 (Completeness) For any $\Sigma \subseteq\{\mathbf{4 , 5}\}$ and any $\Delta$-formula $A$, the following statements are equivalent: 
1. $\mathbf{K} \Sigma^{\Delta} \vdash A$;

2. $\mathbf{K} \Sigma \vdash \operatorname{tr}(A)$;

3. $A$ is valid in all $\mathbf{K} \Sigma$-frames.

Proof: We follow the scheme (1) $\Rightarrow(2) \Leftrightarrow(3) \Rightarrow$ (1). The equivalence (2) $\Leftrightarrow$ (3) is the well-known (cf. [2]) completeness of $\mathbf{K} \Sigma$ with respect to $\mathbf{K} \Sigma$-frames. In the rest of the proof we refer to $\Delta$-formulas as just formulas.

$(1) \Rightarrow(2) \quad$ The axioms of $\mathbf{K}^{\Delta}$ are valid in any frame and so the translations thereof are provable in $\mathbf{K}$. The case of the axiom $\left(\mathrm{A}_{4}^{\Delta}\right)$ is considered in [5]. We give a sketch of a derivation of (the translation of) the axiom $\left(A_{5}^{\Delta}\right)$ in K5:

K5 $\vdash \neg \Delta p \longrightarrow \diamond p \rightarrow \square \diamond p \quad \longrightarrow \square \neg \Delta p \rightarrow \square(q \rightarrow \neg \Delta p) \rightarrow \Delta(q \rightarrow \neg \Delta p)$.

(3) $\Rightarrow$ (1) We construct the canonical model $M_{L}=\left\langle W_{L}, \uparrow, \models\right\rangle$ for the logic $L=$ $\mathbf{K} \Sigma^{\Delta}$. Its worlds are maximal $L$-consistent sets of formulas. A valuation is defined in the usual way: $w \models p \Leftrightarrow p \in w$, for any world $w$ and a variable $p$. Before defining the relation $\uparrow$ we introduce some notation.

For a formula $A$, denote $\nabla A:=\left\{\Delta(B \rightarrow A) \mid B \in \mathbf{F m}^{\Delta}\right\}$. In the subsequent proof, the symbol $\otimes$ plays the role similar to that of $\square$ in the standard canonical model argument for $\square$-logics. The difference is in their "types": the operator $\square$ maps a formula to a formula, whereas $\nabla$ maps a formula to a set of formulas. Note that semantically $\square$ is by no means equivalent to $\square$, in the sense that the truth at a world $w$ of the formula $\square A$ is not equivalent to the truth at $w$ of all formulas in the set $\square A$.

Now denote $\sharp w:=\left\{A \in \mathbf{F m}^{\Delta} \mid \otimes A \subseteq w\right\}$. Finally, put $w \uparrow x$ if and only if $\sharp w \subseteq x$.

Lemma 2.3 For any world $w \in W_{L}$, the following properties are satisfied:

$\mathbf{1}^{\circ}$ (Dichotomy) If $\Delta A \in w$ then either $A \in \sharp w$ or $\neg A \in \sharp w$.

$\mathbf{2}^{\circ}$ The set $\sharp w$ is closed under (even empty) conjunction (hence $\sharp w \neq \varnothing$ ).

$3^{\circ}$ The set $\sharp w$ is closed under derivability in $L$ : if $A \in \sharp w$ and $L \vdash A \rightarrow B$, then $B \in \sharp w$.

$4^{\circ}$ The dichotomy property is reversible: if $A \in \sharp w$ then $\Delta A \in w$.

Proof:

$\mathbf{1}^{\circ}$ Suppose $A, \neg A \notin \sharp w$, then by definition of $\sharp w$, for some formulas $B, C$ we have: $\neg \Delta(B \rightarrow A) \in w, \neg \Delta(C \rightarrow \neg A) \in w$. However, using the dichotomy axiom, we derive: $\mathbf{K}^{\Delta} \vdash \Delta A \rightarrow[\Delta(B \rightarrow A) \vee \Delta(C \rightarrow \neg A)]$, and hence $w$ is even $\mathbf{K}^{\Delta}$-inconsistent, which contradicts our assumptions.

$\mathbf{2}^{\circ}$ By definition, the empty conjunction is $\top$. Since $\Delta(B \rightarrow \top)$ is provable in $\mathbf{K}^{\Delta}$ for any formula $B$, we have $\nabla \top \subseteq \mathbf{K}^{\Delta} \subseteq L \subseteq w$ and so $\top \in \sharp w$.

Now let $A, B \in \sharp w$ and prove that $(A \& B) \in \sharp w$, that is, $\Delta[C \rightarrow(A \& B)] \in w$, for any formula $C$. From $\nabla A \subseteq w$ and $\nabla B \subseteq w$ it follows that $\Delta(C \rightarrow A) \in w$ and $\Delta(C \rightarrow B) \in w$. Using Lemma2.1. we derive

$$
\begin{aligned}
\mathbf{K}^{\Delta} \vdash \Delta(C \rightarrow A) \& \Delta(C \rightarrow B) & \longrightarrow \Delta[(C \rightarrow A) \&(C \rightarrow B)] \longleftrightarrow \\
& \longleftrightarrow \Delta[C \rightarrow(A \& B)] .
\end{aligned}
$$


Since $w$ is closed under conjunction and derivability in $\mathbf{K}^{\Delta}$ (and even in $L$ ), we conclude: $\Delta[C \rightarrow(A \& B)] \in w$.

$3^{\circ}$ To prove that $B \in \sharp w$, we take an arbitrary formula $C$ and show that $\Delta(C \rightarrow$ $B) \in w$. Since $\otimes A \subseteq w$, we have $\Delta[\neg(C \rightarrow B) \rightarrow A] \in w$. The assumption $L \vdash A \rightarrow B$ truth-functionally implies $L \vdash[\neg(C \rightarrow B) \rightarrow A] \leftrightarrow[C \rightarrow B]$, and an application of the rule $\left(\mathrm{RE}^{\Delta}\right)$ finally yields $\Delta(C \rightarrow B) \in w$.

$4^{\circ} \otimes A \subseteq w$ implies $\Delta(\top \rightarrow A) \in w$, which is equivalent to $\Delta A \in w$.

Lemma $2.4(\models=\ni) \quad$ For any formula $A$ and $a$ world $w, w \models A \Leftrightarrow A \in w$.

Proof: By induction on $A$. Atomic and Boolean cases are trivial. Now consider $A=\Delta B$.

$(\Leftarrow)$

$$
\begin{array}{ll}
\Delta B \in w & \left.\Rightarrow \text { (by dichotomy } \mathbf{1}^{\circ}\right) \\
B \in \sharp w \text { or } \neg B \in \sharp w & \Rightarrow \text { (by definition of } \uparrow \text { ) } \\
(\forall x \downarrow w B \in x) \text { or }(\forall x \downarrow w \neg B \in x) & \Rightarrow \text { (by consistency of } x) \\
(\forall x \downarrow w B \in x) \text { or }(\forall x \downarrow w B \notin x) & \Rightarrow \text { (by induction hypothesis) } \\
(\forall x \downarrow w x \models B) \text { or }(\forall x \downarrow w x \not \neq B) & \Rightarrow w \models \Delta B .
\end{array}
$$

$(\Rightarrow)$

Suppose $\Delta B \notin w$. Then the sets $X=\sharp w \cup\{B\}$ and $Y=\sharp w \cup\{\neg B\}$ are $L$-consistent. For, if $Y$ is not then $L \vdash\left(A_{1} \& \cdots \& A_{n}\right) \rightarrow B$ for some formulas $A_{1}, \ldots, A_{n} \in \sharp w$ and $n \geq 0$. By $\mathbf{2}^{\circ},\left(A_{1} \& \cdots \& A_{n}\right) \in \sharp w$, then $B \in \sharp w$ by $\mathbf{3}^{\circ}$ and $\Delta B \in w$ by $\mathbf{4}^{\circ}$, which is not the case. The argument for $X$ is similar except for additional use of the mirror axiom.

Therefore, $X$ and $Y$ are contained in some worlds $x$ and $y$. Since $\sharp w \subseteq x$ and $\sharp w \subseteq y$, we have $w \uparrow x$ and $w \uparrow y$; by induction hypothesis, $B \in x$ and $B \notin y$ imply $x=B$ and $y \not \neq B$, thus $w \not \neq \Delta B$.

By this lemma, the canonical model falsifies all the nontheorems of $L$. To conclude the proof, it remains to check that the canonical frame is a $\mathbf{K} \Sigma$-frame. The case $\Sigma=\varnothing$ is trivial.

Suppose $\mathbf{4} \in \Sigma$ and prove that $\uparrow$ is transitive. Let $w \uparrow x \uparrow y$ and show that $w \uparrow y$, that is, $\sharp w \subseteq y$. Take any $A \in \sharp w$, then $\Delta(B \rightarrow A) \in w$, for every $B$. By the axiom $\left(\mathrm{A}_{\mathbf{4}}^{\Delta}\right), \mathbf{K}^{\Delta} \vdash \Delta(B \rightarrow A) \rightarrow \Delta[C \rightarrow \Delta(B \rightarrow A)]$, for any $C$. Since $w$ is closed under $\mathbf{K} \mathbf{4}^{\Delta}$-derivability, $\Delta[C \rightarrow \Delta(B \rightarrow A)] \in w$. Hence $\nabla \Delta(B \rightarrow A) \subseteq w$ and $\Delta(B \rightarrow A) \in \sharp w \subseteq x$, whence $\nabla A \subseteq x$ and $A \in \sharp x \subseteq y$, as desired.

Suppose $5 \in \Sigma$ and prove that $\uparrow$ is euclidean. Let $w \uparrow x, w \uparrow y$ and show that $x \uparrow y$, that is, $\sharp x \subseteq y$. Take any $A \notin y$, then $A \notin \sharp w$ by $\sharp w \subseteq y$, hence $\neg \Delta(B \rightarrow A) \in w$, for some $B$. Since $w$ is closed under $\mathbf{K 5}^{\Delta}$-derivability, we apply $\left(\mathrm{A}_{\mathbf{5}}^{\Delta}\right)$ to obtain $\Delta[C \rightarrow$ $\neg \Delta(B \rightarrow A)] \in w$, for all $C$, therefore $\bigotimes \neg \Delta(B \rightarrow A) \subseteq w$. By $w \uparrow x$, we conclude: $\neg \Delta(B \rightarrow A) \in x$, thus $\square A \nsubseteq x$ and $A \notin \sharp x$, hence the claim.

3 A problem with seriality It is known (see [2]) that the canonical frame of the logic $\mathbf{D}=\mathbf{K D}$ is serial (and so it validates $\mathbf{D}$ ). It turns out that this does not hold for the $\Delta$ logic of $\mathbf{D}$. More precisely, an application of the construction described in the previous section to $\mathbf{D}$ yields a frame which is not serial. A possible solution of the problem 
consists in appropriate modification of the construction. However, in this section we show that, in order to axiomatize $\Delta$-logic of some logics containing $\mathbf{D}$, this is not necessary. In particular, we prove that $\mathbf{K D} \Sigma^{\Delta}=\mathbf{K} \Sigma^{\Delta}$, for any $\Sigma \subseteq\{\mathbf{4}, \mathbf{5}\}$. Note that the canonical frame of $\mathbf{K D} \Sigma^{\Delta}$ validates $\mathbf{K} \Sigma^{\Delta}$ (since its transitivity and euclideanness follow from the presence of the axioms $\left(\mathrm{A}_{\mathbf{4}}^{\Delta}\right)$ and $\left(\mathrm{A}_{\mathbf{5}}^{\Delta}\right)$ in the logic) and hence $\mathbf{K D} \Sigma^{\Delta}$, so $\mathrm{KD} \Sigma^{\Delta}$ is a canonical logic in the usual sense.

Recall that the logic Ver is obtained by adding to $\mathbf{K}$ the axiom $\square p$, that is, Ver $=$ $\mathbf{K}+\square p$. Similarly, Triv $=\mathbf{K}+(\square p \leftrightarrow p)$. The results obtained in $[7$ imply that, for any logic $L \supseteq \mathbf{K}$, if $\diamond \top \in L$ then $L \subseteq$ Triv, otherwise $L \subseteq$ Ver. Now observe that the logic $\operatorname{Ver}^{\Delta}$ can be axiomatized by adding to $\mathbf{K}^{\Delta}$ the axiom $\Delta p$. The following fact is already mentioned in 4, p. 225.

Theorem 3.1 For any consistent $\square$-logic L containing $\mathbf{K}, L^{\Delta} \subseteq \mathbf{V e r}^{\Delta}$.

Proof: Let $A \in L^{\Delta}$. Consider a formula $A$ as a truth-functional compound of variables and formulas of the form $\Delta B: A=f\left(\vec{p}, \Delta B_{1}, \ldots, \Delta B_{n}\right)$, where $f$ is a nonmodal formula and $\vec{p}$ is the list of all variables occurring in $A$. We must show that $\operatorname{Ver}^{\Delta} \vdash A$, or equivalently, $\operatorname{Ver}^{\Delta} \vdash f(\vec{p}, \top, \ldots, \top)$, since $\operatorname{Ver}^{\Delta} \vdash \Delta B \leftrightarrow \top$ for any $B$. The logic $\operatorname{Ver}^{\Delta}$ is conservative over propositional logic, so it remains to prove that $f(\vec{p}, \top, \ldots, \top)$ is a tautology. To this end, we take an arbitrary $\vec{\sigma} \in\{\perp, \top\}^{m}$ with $m=|\vec{p}|$ and check that the value $\vartheta:=f(\vec{\sigma}, \top, \ldots, \top)=\top$.

Since $L^{\Delta}$ is closed under the rule (Sub), substituting $\vec{\sigma}$ for $\vec{p}$ in $A$ yields a $\Delta$ sentence (i.e., $\Delta$-formula containing no variable) $A[\vec{\sigma} / \vec{p}] \in L^{\Delta}$. It is easily seen that any $\Delta$-sentence of the form $\Delta B$ is equivalent to $\top$ in $\mathbf{K}^{\Delta}$ (for this, observe that any $\Delta$-sentence is equivalent to either $\perp$ or $T$, and both $\Delta \perp$ and $\Delta T$ are equivalent to T). Hence $A[\vec{\sigma} / \vec{p}]$ is equivalent to $\vartheta$ in $\mathbf{K}^{\Delta}$, so $\vartheta \in L^{\Delta}$. Finally, due to consistency of $L^{\Delta}$, we conclude: $\vartheta=\top$.

Corollary 3.2 For any consistent $\square$-logic $L \supseteq \mathbf{D}$, the canonical frame for $L^{\Delta}$ is not serial and hence not an L-frame.

Proof: The set $\operatorname{Ver}^{\Delta}$ is $L^{\Delta}$-consistent, since it is even $\mathbf{V e r}^{\Delta}$-consistent and $L^{\Delta} \subseteq$ $\operatorname{Ver}^{\Delta}$. So it is contained in some world $w$ of the canonical frame $F_{L}$ for $L^{\Delta}$. We claim that $w$ has no $\uparrow$-successors (and thus the frame $F_{L}$ is not serial). Indeed, if $w \uparrow x$ then $\sharp w \subseteq x$, but $w$ contains $\Delta A$ for all formulas $A$, hence $\sharp w=\mathbf{F m}^{\Delta}$ and $x$ is inconsistent.

Now we show, following 4 , that adding the axiom $\left(A_{D}^{\square}\right)$ to some $\square$-logics does not change $\Delta$-logic thereof. Let $F=\langle W, \uparrow\rangle$ be a frame. A set of worlds accessible from $w \in W$ is denoted by $w \uparrow:=\{x \in W \mid w \uparrow x\}$. We turn each "blind" world into a world "seeing" only itself and obtain a frame $\widehat{F}:=\langle W, \uparrow\rangle$, where $\Uparrow:=\uparrow \cup\{\langle w, w\rangle \mid$ $w \uparrow=\varnothing\}$. Given a class of frames $\mathcal{F}$, we denote $\widehat{\mathcal{F}}:=\{\widehat{F} \mid F \in \mathcal{F}\}$. In 4 it is noted that frames $F$ and $\widehat{F}$ validate the same $\Delta$-formulas. Consequently, the $\Delta$-logic of classes $\mathcal{F}$ and $\widehat{\mathcal{F}}$ coincide.

Definition 3.3 We call the set $\mathcal{L}^{\square}(\mathcal{F}):=\left\{A \in \mathbf{F m}^{\square} \mid \mathcal{F} \models A\right\}$ a $\square$-logic of a class of frames $\mathcal{F}$. A $\square$-logic $L$ is called (Kripke) complete (with respect to a class $\mathcal{F}$ ) if $L=\mathcal{L}^{\square}(\mathcal{F})$; finitely approximable if it is complete with respect to a class of finite frames. For the $\Delta$-language, the same notions are defined similarly. 
Theorem 3.4 Suppose $a \square$-logic $L$ is complete with respect to a class $\mathcal{F}$ and $L D$ is the smallest logic containing $L$ and the axiom $\left(\mathrm{A}_{\mathbf{D}}^{\square}\right)$. If $\widehat{\mathcal{F}} \subseteq \mathcal{F}$ then $L \mathbf{D}^{\Delta}=L^{\Delta}$.

Proof: The inclusion ( $\supseteq$ ) is trivial. Now take any $A \in L \mathbf{D}^{\Delta}$; clearly, $A \in L^{\Delta} \Leftrightarrow$ $\operatorname{tr}(A) \in L \Leftrightarrow \mathcal{F} \models \operatorname{tr}(A) \Leftrightarrow \mathcal{F} \models A$, so it remains to show that $F \models A$, for any frame $F \in \mathcal{F}$. Since $\widehat{\mathcal{F}} \subseteq \mathcal{F}$, we have $\widehat{F} \in \mathcal{F}$ and so $\widehat{F} \models L$; besides, $\widehat{F}$ is serial, hence $\widehat{F} \models\left(\mathrm{A}_{\mathbf{D}}^{\square}\right)$. Thus $\widehat{F} \models L \mathbf{D}$, whence $\widehat{F} \models L \mathbf{D}^{\Delta}$, in particular, $\widehat{F} \models A$. By the above, this is equivalent to $F \models A$.

As a consequence, $\mathbf{K D} \Sigma^{\Delta}=\mathbf{K} \Sigma^{\Delta}$, for any $\Sigma \subseteq\{\mathbf{4}, \mathbf{5}\}$, since the transitivity and euclideanness properties are preserved as we pass from $F$ to $\widehat{F}$. For the case $\Sigma=\varnothing$ the result was obtained in [4].

4 Definability Insofar as the $\Delta$-language is embeddable into the $\square$-language via the translation tr, the expressive power of the former is no more than that of the latter. Moreover, as is already noted in [4], it is essentially less, for some well-known $\square$-definable classes are not $\Delta$-definable. In this section we show that this effect is explainable by the fact that every $\Delta$-definable class of frames must contain the class of functional frames.

Definition 4.1 A class of frames $\mathcal{F}$ is $\square$-definable if there exists a set $\Gamma$ of $\square$ formulas such that, for any frame $F, F \in \mathcal{F} \Leftrightarrow F \models \Gamma$; in this case $\Gamma$ is said to $\square$ define $\mathcal{F}$. For the $\Delta$-language, the same notions are defined similarly.

Following [1], p. 91, we call a frame functional if it satisfies the condition

$$
\forall w \forall x \downarrow w \forall y \downarrow w \quad x=y \quad \text { (functionality). }
$$

In 44 it is shown that the logic $\operatorname{Ver}^{\Delta}$ is complete with respect to the class $\mathfrak{F u n c}$ of functional frames and moreover, this logic $\Delta$-defines the class $\mathfrak{F u n c}$.

Lemma 4.2 If $\Gamma$ (respectively, $\Gamma^{\prime}$ ) defines a class $\mathcal{F}$ (respectively, $\mathcal{F}^{\prime}$ ) and $\Gamma \subseteq \Gamma^{\prime}$ then $\mathcal{F}^{\prime} \subseteq \mathcal{F}$ (of course, $\Gamma$ and $\Gamma^{\prime}$ are supposed to be in the same, $\square$ - or $\Delta$-, language).

Proof: By assumption, $\forall F(F \in \mathcal{F} \Leftrightarrow F \models \Gamma)$ and $\forall F\left(F \in \mathcal{F}^{\prime} \Leftrightarrow F \models \Gamma^{\prime}\right)$. Then, for any frame $F$, we have $F \in \mathcal{F}^{\prime} \Leftrightarrow F \models \Gamma^{\prime} \Rightarrow F \models \Gamma \Leftrightarrow F \in \mathcal{F}^{\prime}$.

Theorem 4.3 If a class of frames $\mathcal{F} \neq \varnothing$ is $\Delta$-definable then $\mathfrak{F u n c} \subseteq \mathcal{F}$.

Proof: $\quad$ Suppose a set $\Gamma \Delta$-defines $\mathcal{F}$. Take a logic $L:=\mathcal{L}^{\square}(\mathcal{F})$. Since $\mathcal{F} \models \Gamma$, we have $\mathcal{F} \models \operatorname{tr}(\Gamma)$, whence $\operatorname{tr}(\Gamma) \subseteq L$ and $\Gamma \subseteq L^{\Delta}$. By Theorem 3.1. $L^{\Delta} \subseteq \operatorname{Ver}^{\Delta}$, so $\Gamma \subseteq \operatorname{Ver}^{\Delta}$. Now Lemma 4.2 implies $\mathfrak{F u n c} \subseteq \mathcal{F}$.

Corollary 4.4 The classes of reflexive, serial, transitive, symmetric, euclidean frames, as well as any subclass thereof, are not $\Delta$-definable.

This corollary (for the first four classes) was already obtained by Humberstone [4, Theorem 4.2 from other considerations.

We shall return to definability issues in the next section. Now we show that the map $L \mapsto L^{\Delta}$ preserves (Kripke) completeness; moreover, it is an epimorphism of 
the lattice (with respect to inclusion) of complete logics containing $\mathbf{K}$ onto the lattice of complete logics in the segment $\left[\mathbf{K}^{\Delta}, \mathbf{V e r}^{\Delta}\right]$. In addition, we obtain the same result for finitely approximable logics.

\section{Theorem 4.5}

(a) If a $\square$-logic $L$ is complete with respect to a class $\mathcal{F}$ then $L^{\Delta}$ is complete with respect to the same class $\mathcal{F}$. In particular, if $L$ is finitely approximable then so is $L^{\Delta}$.

(b) If a $\Delta$-logic $M$ is complete with respect to a class $\mathcal{F}$ then $M=L^{\Delta}$ for some $\square$-logic L that is complete with respect to the same class $\mathcal{F}$. In particular, if $M$ is finitely approximable then $M=L^{\Delta}$ for some finitely approximable logic $L$.

Proof:

(a) By assumption, $L=\mathcal{L}^{\square}(\mathcal{F})$; then $L^{\Delta}=\mathcal{L}^{\Delta}(\mathcal{F})$, since, for any $\Delta$-formula $A$, $A \in \mathcal{L}^{\Delta}(\mathcal{F}) \Leftrightarrow \mathcal{F}=A \Leftrightarrow \mathcal{F} \models \operatorname{tr}(A) \Leftrightarrow \operatorname{tr}(A) \in L \Leftrightarrow A \in L^{\Delta}$.

(b) Suppose $M=\mathcal{L}^{\Delta}(\mathcal{F})$. Then take $L:=\mathcal{L}^{\square}(\mathcal{F})$ and the above argument shows that $M=L^{\Delta}$.

The above map is not injective, as the following example shows. Clearly, Triv $\neq$ Ver, however, $\operatorname{Triv}^{\Delta}=\operatorname{Ver}^{\Delta}$, since $\operatorname{Triv}^{\Delta} \vdash \Delta p$ and $\mathbf{V e r}^{\Delta}$ is the greatest noncontingency logic, by Theorem 3.1 . The same argument applied to any logic $L$ containing the functionality axiom $\diamond p \rightarrow \square p$ yields $L^{\Delta}=\mathbf{V e r}^{\Delta}$.

On the other hand, the restriction of this map to the family of logics containing $\mathbf{T}$ is injective. This is an easy consequence of $\Delta$-definability of $\square$ in these logics by $\square A=A \& \Delta A$ and hence the existence of a natural translation from $\square$ - into $\Delta$ language. Details are left to the reader.

We close this section with an observation that adding the class $\mathfrak{F u n c}$ to a nonempty class $\mathcal{F}$ does not change its $\Delta$-logic. Thus, any (consistent) complete $\Delta$-logic can be represented as the $\Delta$-logic of a class containing $\mathfrak{F u n c}$.

Lemma 4.6 $\mathcal{L}^{\Delta}(\mathcal{F})=\mathcal{L}^{\Delta}(\mathcal{F} \cup \mathfrak{F u n c})$ for any nonempty class $\mathcal{F}$.

Proof: First, $\mathcal{L}^{\Delta}(\mathcal{F} \cup \mathcal{G})=\mathcal{L}^{\Delta}(\mathcal{F}) \cap \mathcal{L}^{\Delta}(\mathcal{G})$, for any classes $\mathcal{F}$ and $\mathcal{G}$. Secondly, since $\mathcal{F}$ is nonempty, the logic $L:=\mathcal{L}^{\square}(\mathcal{F})$ is consistent and $L^{\Delta} \subseteq \mathbf{V e r}^{\Delta}$ by Theorem 3.1. Thirdly, the argument used in the proof of Theorem 4.5 a) yields $\mathcal{L}^{\Delta}(\mathcal{F})=$ $L^{\Delta}$ and therefore $\mathcal{L}^{\Delta}(\mathcal{F}) \subseteq \operatorname{Ver}^{\Delta}$. Finally, $\mathcal{L}^{\Delta}(\mathcal{F} \cup \mathfrak{F} \mathfrak{u n c})=\mathcal{L}^{\Delta}(\mathcal{F}) \cap \mathcal{L}^{\Delta}(\mathfrak{F u n c})=$ $\mathcal{L}^{\Delta}(\mathcal{F}) \cap \operatorname{Ver}^{\Delta}=\mathcal{L}^{\Delta}(\mathcal{F})$.

5 First-order correspondence Here we present first-order formulas characterizing the same classes of frames as the axioms of the noncontingency logics mentioned in Section 2. We begin with introducing some convenient notation.

Validity of a first-order formula $\varphi$ in a frame $F=\langle W, \uparrow\rangle$ will be denoted by $F \models \varphi$ to distinguish from validity of modal formulas. Quantification over worlds accessible from a given world $w \in W$ will be written as $\forall x \downarrow w$ and $\exists x \downarrow w$ (as was done in Section 2). Instead of $X \cap Y \neq \varnothing$, we write briefly $X \cap Y$. 
A world $w$ is called functional if it "sees" at most one world; this property is obviously first-order expressible by

$$
F n c(w) \leftrightharpoons \forall x, y \downarrow w(x=y) .
$$

A world $w$ is branching ( $\operatorname{Bra}(w)$, in symbols) if it is not functional. We introduce special notation for bounded quantification over branching worlds:

$$
\begin{aligned}
& \widehat{\forall} w \varphi(w) \leftrightharpoons \forall w[\operatorname{Bra}(w) \rightarrow \varphi(w)] ; \\
& \widehat{\exists} w \varphi(w) \leftrightharpoons \exists w[\operatorname{Bra}(w) \& \varphi(w)] .
\end{aligned}
$$

Bounded quantification over branching worlds accessible from $w$ is written as

$$
\begin{aligned}
& \widehat{\forall} x \downarrow w \varphi(x) \leftrightharpoons \forall x[\{w \uparrow x \& \operatorname{Bra}(x)\} \rightarrow \varphi(x)] ; \\
& \widehat{\exists} x \downarrow w \varphi(x) \leftrightharpoons \exists x[w \uparrow x \& \operatorname{Bra}(x) \& \varphi(x)] .
\end{aligned}
$$

The existence of a branching world accessible from $w$ will be abbreviated as $\widehat{\exists} x \downarrow w$, which is equivalent to $\widehat{\exists} \downarrow \downarrow w \top$. Finally, by $\mathfrak{T r a n}$ and $\mathfrak{E} \mathfrak{u c l}$ we denote the classes of transitive and euclidean frames, respectively.

Recall that the formula $\Delta p$ defines the class $\mathfrak{F u n c}$ of frames satisfying the condition $\forall w F n c(w)$. The main theorem of this section states that the classes of frames defined by $\Delta$-formulas,

$$
\begin{array}{ll}
\left(\mathrm{A}_{\mathrm{T}}^{\Delta}\right) & p \rightarrow[\Delta(p \rightarrow q) \rightarrow(\Delta p \rightarrow \Delta q)] \\
\left(\mathrm{A}_{4}^{\Delta}\right) & \Delta p \rightarrow \Delta(q \rightarrow \Delta p) \\
\left(\mathrm{A}_{4 b}^{\Delta}\right) & \Delta p \rightarrow \Delta \Delta p \\
\left(\mathrm{~A}_{\mathbf{5}}^{\Delta}\right) & \neg \Delta p \rightarrow \Delta(q \rightarrow \neg \Delta p) \\
\left(\mathrm{A}_{\mathbf{5}^{\prime}}^{\Delta}\right) & \Delta(\Delta p \rightarrow p) \\
\left(\mathrm{A}_{\mathbf{5} b}^{\Delta}\right) & \Delta \Delta p,
\end{array}
$$

are first-order definable by the following formulas (braces enclosing two formulas mean a conjunction thereof):

$$
\begin{array}{ll}
\left(\varphi_{\mathbf{T}}^{\Delta}\right) & \widehat{\forall} w \quad w \uparrow w \\
\left(\varphi_{\mathbf{4}}^{\Delta}\right) & \widehat{\forall} w \widehat{\forall} x \downarrow w \forall y \downarrow x \quad w \uparrow y \\
\left(\varphi_{\mathbf{4} b}^{\Delta}\right) & \widehat{\forall} w\left[\widehat{\forall} x \downarrow w(x \uparrow \subseteq w \uparrow) \vee \forall x, y \downarrow w\left\{\begin{array}{l}
x \uparrow \backslash w \uparrow=y \uparrow \backslash w \uparrow \\
x \uparrow \cap w \uparrow \Leftrightarrow y \uparrow \cap w \uparrow
\end{array}\right]\right. \\
\left(\varphi_{\mathbf{5}}^{\Delta}\right) & \widehat{\forall} w \forall x, y \downarrow w \quad x \uparrow y \\
\left(\varphi_{\mathbf{5}^{\prime}}^{\Delta}\right) & \widehat{\forall} w \forall x, y \downarrow w \quad x \uparrow y \\
\left(\varphi_{\mathbf{5} b}^{\Delta}\right) & \widehat{\forall} w[\widehat{\exists} x \downarrow w \rightarrow \forall x, y \downarrow w(x \uparrow=y \uparrow)] .
\end{array}
$$

Theorem 5.1 $F \models\left(\mathrm{A}_{\mathfrak{S}}^{\Delta}\right) \Longleftrightarrow F \models\left(\varphi_{\mathfrak{S}}^{\Delta}\right)$, for each $\mathfrak{S} \in\left\{\mathbf{T}, \mathbf{4}, \mathbf{4} b, \mathbf{5}, \mathbf{5} b, \mathbf{5}^{\prime}\right\}$ and any frame $F$.

Proof: Note that the claim for $\mathfrak{S}=\mathbf{T}$ is already stated in [4]. Each formula $\left(\mathrm{A}_{\mathfrak{S}}^{\Delta}\right)$ under consideration belongs to the logic $\mathbf{V e r}^{\Delta}$ and hence is true at any functional world under any valuation. Wherefore in the ' $\Leftarrow$ ' part of our proof it is assumed that we 
are given an arbitrary frame $F=\langle W, \uparrow\rangle$ such that $F \models\left(\varphi_{\mathfrak{S}}^{\Delta}\right)$, a valuation $\models$, and a branching world $w \in W$.

$\mathfrak{S}=\mathbf{T}:$

$(\Rightarrow) \quad$ Assume that $F \not 1 \neq\left(\varphi_{\mathbf{T}}^{\Delta}\right)$, that is, $\widehat{\exists} w \neg(w \uparrow w)$ and then $\exists x, y \downarrow w: x \neq y$. To falsify ( $\mathrm{A}_{\mathrm{T}}^{\Delta}$ ) at $w$, put $p$ (respectively, $q$ ) to be true only at $w$ (respectively, $x$ ). Then $w \models p ; w \models \Delta p$, even $w \models \square \neg p$, since $\forall t \downarrow w t \not \models p ; w \models \Delta(p \rightarrow q)$, even $w \models \square(p \rightarrow q)$, since $\forall t \downarrow w t \models p \rightarrow q$; however, $w \not \models \Delta q$, since $x \models q$ and $y \not \neq q$.

$(\Leftarrow) \quad$ Suppose $w \models p, \Delta p$; by $\left(\varphi_{\mathbf{T}}^{\Delta}\right)$, we have $w \uparrow w$, hence $w \models \square p$. Further, suppose $w \models \Delta(p \rightarrow q)$. To see that $w \models \Delta q$, consider two cases:

(a) $w \models \square(p \rightarrow q)$; together with $w \models \square p$, this implies $w \models \square q$.

(b) $w \models \square \neg(p \rightarrow q)$, that is, $w \models \square(p \& \neg q)$; it follows that $w \models \square \neg q$.

$\mathfrak{S}=\mathbf{4}:$

It suffices to consider a formula $\square p \rightarrow \Delta(q \rightarrow \Delta p)$ instead of $\left(\mathrm{A}_{4}^{\Delta}\right)$.

$(\Rightarrow) \quad$ Assume that $F \not \neq\left(\varphi_{4}^{\Delta}\right)$, that is, $\widehat{\exists} w \widehat{\exists} x \downarrow w \exists y \downarrow x: \neg(w \uparrow y)$. Let $p$ be false only at $y$ and $q$ be true only at $x$. Then $w \models \square p$, since $\neg(w \uparrow y)$; however, $w \not \models$ $\Delta(q \rightarrow \Delta p)$, for the following hold:

(a) $w \models \diamond(q \rightarrow \Delta p)$, since $\operatorname{Bra}(w)$ implies $\exists z \downarrow w, z \neq x$, so that $z \not \neq q$ and $z \models$ $q \rightarrow \Delta p$.

(b) $w \models \diamond \neg(q \rightarrow \Delta p)$, since $w \uparrow x, x \models q$, and $x \not \neq \Delta p$; to see the latter, note that $x \uparrow y$ and $y \not \models p$, whereas $\operatorname{Bra}(x)$ implies $\exists t \downarrow x, t \neq y$, so that $t \models p$.

$(\Leftarrow) \quad$ Suppose $w \models \square p$ and prove that even $w \models \square(q \rightarrow \Delta p)$. Take any $x \downarrow w$; if $\operatorname{Fnc}(x)$ then $x \models \Delta p$ and so $x \models q \rightarrow \Delta p$; if $\operatorname{Bra}(x)$ then even $x \models \square p$, since, for any $y \downarrow x$, we have $w \uparrow y$ by $\left(\varphi_{4}^{\Delta}\right)$, and from $w \models \square p$ it follows that $y \models p$.

$\mathfrak{S}=4 \mathrm{~b}:$

Clearly, instead of $\left(\mathrm{A}_{\mathbf{4} b}^{\Delta}\right)$ we can deal with $\square p \rightarrow \Delta \Delta p$.

$(\Rightarrow) \quad$ Assume that $F \not \neq\left(\varphi_{4 b}^{\Delta}\right)$, that is, $\widehat{\exists} w \widehat{\exists} x_{0} \downarrow w: x_{0} \uparrow \nsubseteq w \uparrow$ and $\exists x, y \downarrow w$ such that at least one of the conditions in braces in $\left(\varphi_{\mathbf{4} b}^{\Delta}\right)$ fails. There are two cases:

1. Either $x$ or $y$ is functional, say, $\operatorname{Fnc}(y)$. Since $\operatorname{Bra}\left(x_{0}\right)$ and $x_{0} \uparrow \nsubseteq \subseteq w \uparrow$, we have $\exists s, t \downarrow x_{0}: s \neq t, \neg(w \uparrow s)$. Let $p$ be false only at $s$. Then $w \models \square p$, for $\neg(w \uparrow s)$; $y \models \Delta p$, for $F n c(y)$; and $x_{0} \not \neq \Delta p$, for $s \not \neq p$ and $t \models p$; thus $w \not \neq \Delta \Delta p$.

2. Both $x$ and $y$ are branching. Then two subcases are possible:

(a) $(x \uparrow \backslash w \uparrow) \neq(y \uparrow \backslash w \uparrow)$. Due to symmetry, we can assume that $\exists s \in$ $(x \uparrow \backslash w \uparrow), s \notin(y \uparrow \backslash w \uparrow)$. This implies $x \uparrow s, \neg(y \uparrow s)$, and $\neg(w \uparrow s)$. Let $p$ be false only at $s$. Then $w \models \square p$, for $\neg(w \uparrow s) ; y \models \Delta p$, even $y \models \square p$, for $\neg(y \uparrow s)$; and $x \not \not \Delta p$, since $s \not=p$, whereas $\operatorname{Bra}(x)$ implies $\exists t \downarrow x$, $t \neq s$, so that $t \models p$; thus $w \not \models \Delta \Delta p$.

(b) Now assume that $(x \uparrow \backslash w \uparrow)=(y \uparrow \backslash w \uparrow)$ and (due to symmetry) $x \uparrow \cap w \uparrow$ and $y \uparrow \cap w \uparrow=\varnothing$. Let $p$ be true only at worlds accessible from $w$. Then $w \models \square p$ by construction; $y \models \Delta p$, even $y \models \square \neg p$, for $y \uparrow \cap w \uparrow=\varnothing$; 
and $x \not \neq \Delta p$, since $x \uparrow \cap w \uparrow$ implies $x \models \diamond p$, whereas $(x \uparrow \backslash w \uparrow)=(y \uparrow \backslash$ $w \uparrow) \neq \varnothing$ (the inequality is due to $\operatorname{Bra}(y)$ and $y \uparrow \cap w \uparrow=\varnothing)$ implies $x \models \diamond \neg p$. Thus again $w \not \models \Delta \Delta p$.

$(\Leftarrow) \quad$ According to $\left(\varphi_{\mathbf{4} b}^{\Delta}\right)$, two cases are possible:

1. $\widehat{\forall} x \downarrow w(x \uparrow \subseteq w \uparrow)$. Then even $w \models \square p \rightarrow \square \Delta p$, for assume that $w \models \square p$ and $w \uparrow x$. If $F n c(x)$ then $x \models \Delta p$; if $\operatorname{Bra}(x)$ then even $x \models \square p$, since $x \uparrow \subseteq w \uparrow$.

2. Now suppose the second disjunct in $\left(\varphi_{\mathbf{4} b}^{\Delta}\right)$ holds. We must show that $w \models$ $\square p \& \diamond \neg \Delta p \rightarrow \square \neg \Delta p$. Assume that $w \models \square p \& \diamond \neg \Delta p$, then $\exists x \downarrow w: x \not \mid$ $\Delta p$, hence $\exists s, t \downarrow x: s \models p, t \not \neq p$ (clearly, $s \neq t$ ), and so $\operatorname{Bra}(x)$. Now take any $y \downarrow w$; to see that $y \not \models \Delta p$, first note that $\neg(w \uparrow t)$, for $w \models \square p$, hence $t \in(x \uparrow \backslash w \uparrow)=(y \uparrow \backslash w \uparrow)$, so $y \uparrow t$ and $y \models \diamond \neg p$; secondly, consider cases:

(a) $w \uparrow s$; then $x \uparrow \cap w \uparrow$, hence $y \uparrow \cap w \uparrow$ by $\left(\varphi_{\mathbf{4} b}^{\Delta}\right)$ and so $y \models \diamond p$.

(b) $\neg(w \uparrow s)$; then $s \in(x \uparrow \backslash w \uparrow)=(y \uparrow \backslash w \uparrow)$ (the equality holds by $\left.\left(\varphi_{\mathbf{4 b}}^{\Delta}\right)\right)$, whence $y \uparrow s$ and $y \models \diamond p$.

$\mathfrak{S}=\mathbf{5}:$

$(\Rightarrow) \quad$ Assume that $F \not \neq\left(\varphi_{\mathbf{5}}^{\Delta}\right)$, that is, $\widehat{\exists} w \exists x, y \downarrow w: \neg(x \uparrow y)$. From $\operatorname{Bra}(w)$ it follows that $\exists z \downarrow w: z \neq x$ (possibly $z=y$ ). Let $p$ (respectively, $q$ ) be true only at $y$ (respectively, $x$ ). Then $w \models \neg \Delta p$, since $y \models p$ and either $x$ or $z$ differs from $y$, call it $t$, so that $w \uparrow t$ and $t \not \models p ; w \models \diamond(q \rightarrow \neg \Delta p)$, for $z \not \models q ; w \models \diamond \neg(q \rightarrow \neg \Delta p)$, since $x \models q$ and $x \models \Delta p$, even $x=\square \neg p$, for $\neg(x \uparrow y)$.

$(\Leftarrow) \quad$ Suppose $w \models \neg \Delta p$, then $\exists x, y \downarrow w: x \models p, y \not \models p$. We prove that even $w \models$ $\square(q \rightarrow \neg \Delta p)$ and moreover: $w \models \square \neg \Delta p$. For any $z \downarrow w$, we have $z \uparrow x, z \uparrow y$ by $\left(\varphi_{5}^{\Delta}\right)$, hence $z \models \diamond p$ and $z \models \diamond \neg p$ and so $z=\neg \Delta p$.

$\mathfrak{S}=\mathbf{5}^{\prime}$ :

The formula $\left(\mathrm{A}_{\mathbf{5}^{\prime}}^{\Delta}\right)$ is equivalent (modulo replacing $\neg p$ by $\left.p\right)$ to $\diamond(p \& \Delta p) \rightarrow(\square p \&$ $\square \Delta p)$.

$(\Rightarrow) \quad$ Assume that $F \not 1\left(\varphi_{\mathbf{5}^{\prime}}^{\Delta}\right)$, that is, $\widehat{\exists} w \exists x, y \downarrow w: \neg(x \uparrow y)$. Consider two cases:

1. $x=y$. Let $p$ be true only at $x$. Then $w \models \diamond(p \& \Delta p)$, since $x \models p$ and $x \models \square \neg p$, for $\neg(x \uparrow x)$; however, $w \not \neq \square p$, since $\operatorname{Bra}(w)$ implies $\exists z \downarrow w: z \neq$ $x$, so that $z \not \neq p$.

2. $x \neq y$. Let $p$ be false only at $y$. Then $w \models \diamond(p \& \Delta p)$, since $x \models p$ and $x \models \square p$, for $\neg(x \uparrow y)$; however, $w \not \models \square p$, since $y \not \neq p$.

$(\Leftarrow) \quad$ Suppose $w \models \diamond(p \& \Delta p)$. Then $\exists x \downarrow w: x \models p$ and $x \models \Delta p$. From $\left(\varphi_{\mathbf{5}^{\prime}}^{\Delta}\right)$ it follows that $w \uparrow \subseteq x \uparrow$. Since $w \uparrow x$, we have $x \uparrow x$, whence $x \models \square p$. Therefore $w \models \square p$, since $w \uparrow \subseteq x \uparrow$; and for any $y \downarrow w$ we have $y \models \Delta p$ and even $y \models \square p$; to see the latter, first note that $w \uparrow x$ and $w \uparrow y$ imply $y \uparrow x$; now take any $z \downarrow y$. If $z=x$ then $z=p$; if $z \neq x$ then from $y \uparrow x, y \uparrow z$, and hence $\operatorname{Bra}(y)$ we infer, by $\left(\varphi_{\mathbf{5}^{\prime}}^{\Delta}\right)$, that $x \uparrow z$, so $z \models p$. 
$\mathfrak{S}=\mathbf{5} b:$

The formula $\left(\mathrm{A}_{\mathbf{5} b}^{\Delta}\right)$ is equivalent to $\diamond \Delta p \rightarrow \square \Delta p$.

$(\Rightarrow) \quad$ Assume that $F \not \neq\left(\varphi(\Delta)\right.$, that is, $\widehat{\exists} w \widehat{\exists} x_{0} \downarrow w$ and $\exists x, y \downarrow w: x \uparrow \neq y \uparrow$. Then two cases are possible:

1. Either $x$ or $y$ is functional, say, $\operatorname{Fnc}(y)$. Since $\operatorname{Bra}\left(x_{0}\right)$, we have $\exists s, t \downarrow x_{0}: s \neq t$. Let $p$ be true only at $s$. Then $w \models \diamond \Delta p$, for $y \models \Delta p$ by $F n c(y)$; and $w \models$ $\diamond \neg \Delta p$, since $x_{0} \not \models \Delta p$, for $s \models p$ and $t \not \models p$. Thus $w \not \models \Delta \Delta p$.

2. Both $x$ and $y$ are branching. Since $x \uparrow \neq y \uparrow$, we can assume (due to symmetry) that $\exists s \downarrow x: \neg(y \uparrow s)$. Let $p$ be true only at $s$. Then $w \models \diamond \Delta p$, for $y \models \Delta p$ and even $y \models \square p$ by virtue of $\neg(y \uparrow s)$; and $w \models \diamond \neg \Delta p$, since $x \not \models \Delta p$ : indeed, $s \models p$, whereas $\operatorname{Bra}(x)$ implies $\exists t \downarrow x: t \neq s$, hence $t \not \neq p$. Again $w \not \neq \Delta \Delta p$.

$(\Leftarrow) \quad$ There are two possibilities:

1. $\forall x \downarrow w F n c(x)$. Then $\forall x \downarrow w x \models \Delta p$, hence $w \models \square \Delta p$ and so $w \models\left(\mathrm{A}_{\mathbf{5} b}^{\Delta}\right)$.

2. $\widehat{\exists} x \downarrow w$. Assume that $w \models \diamond \Delta p$, that is, $\exists x \downarrow w: x \models \Delta p$. Take any $y \downarrow w$; then $x \uparrow=y \uparrow$ by $\left(\varphi_{\mathbf{5} b}^{\Delta}\right)$, whence $y \models \Delta p$. Thus $w \models \diamond \Delta p \rightarrow \square \Delta p$.

Corollary 5.2 The following strict inclusions hold. Other inclusions between these classes of frames follow from the exhibited ones.

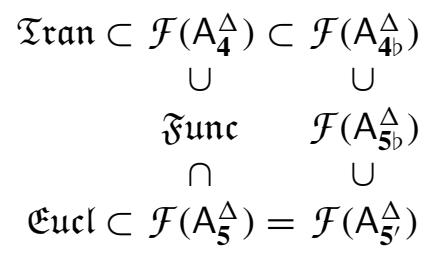

Proof: That $\mathfrak{F u n c} \subseteq \mathcal{F}(A)$ for any $\Delta$-formula $A$ is proved in Theorem 4.3. The Completeness Theorem 2.2 implies that $\mathfrak{T} \mathfrak{r a n} \subseteq \mathcal{F}\left(\mathrm{A}_{4}^{\Delta}\right)$ as well as $\mathfrak{E} \mathfrak{u} \mathfrak{l} \subseteq \mathcal{F}\left(\mathrm{A}_{5}^{\Delta}\right)$. Since putting $q:=\top$ in $\left(\mathrm{A}_{4}^{\Delta}\right)$ yields a formula equivalent to $\left(\mathrm{A}_{\mathbf{4} b}^{\Delta}\right)$ in $\mathbf{K}^{\Delta}$, we have $\mathcal{F}\left(\mathrm{A}_{\mathbf{4}}^{\Delta}\right) \subseteq \mathcal{F}\left(\mathrm{A}_{\mathbf{4} b}^{\Delta}\right)$. The inclusion $\mathcal{F}\left(\mathrm{A}_{\mathbf{5} b}^{\Delta}\right) \subseteq \mathcal{F}\left(\mathrm{A}_{\mathbf{4} b}^{\Delta}\right)$ is trivial, since $\left(\mathrm{A}_{\mathbf{5} b}^{\Delta}\right)$ is the succedent of the implication in $\left(\mathrm{A}_{\mathbf{4} b}^{\Delta}\right)$. The equality $\mathcal{F}\left(\mathrm{A}_{\mathbf{5}}^{\Delta}\right)=\mathcal{F}\left(\mathrm{A}_{\mathbf{5}^{\prime}}^{\Delta}\right)$ is established in Theorem 5.1.

Let us prove that $\mathcal{F}\left(\mathrm{A}_{5}^{\Delta}\right) \subseteq \mathcal{F}\left(\mathrm{A}_{5 \mathrm{~b}}^{\Delta}\right)$. Suppose $F \models\left(\varphi_{5}^{\Delta}\right)$, that is, any two worlds accessible from a branching world "see" each other (themselves as well). We prove that even $\widehat{\forall} w \forall x, y \downarrow w(x \uparrow=y \uparrow)$. Take a branching world $w$ in $F$ and any $x, y \downarrow w$, then $x \uparrow y$ and $y \uparrow y$. To prove the inclusion $x \uparrow \subseteq y \uparrow$, take any $z \downarrow x$. If $z=y$ then clearly $y \uparrow z$. If $z \neq y$ then from $x \uparrow y$ and $x \uparrow z$ it follows that $\operatorname{Bra}(x)$ and again $y \uparrow z$. The converse inclusion is left to the reader.

The inclusion of classes $\mathfrak{F} \mathfrak{u n} \mathfrak{c}, \mathfrak{T} \mathfrak{r a n}$, and $\mathfrak{E} \mathfrak{u c l}$ into the nearest classes in our diagram is strict, since these three classes are incomparable. The inclusion $\mathcal{F}\left(\mathrm{A}_{\mathbf{5} b}^{\Delta}\right) \subset$ $\mathcal{F}\left(\mathrm{A}_{\mathbf{4} b}^{\Delta}\right)$ is strict, for the following inclusion of logics is strict:

$$
\mathbf{T}^{\Delta}+\left(\mathrm{A}_{\mathbf{4} b}^{\Delta}\right)=\mathbf{S 4}^{\Delta} \subset \mathbf{S 5} \mathbf{5}^{\Delta}=\mathbf{T}^{\Delta}+\left(\mathrm{A}_{\mathbf{5} b}^{\Delta}\right) .
$$




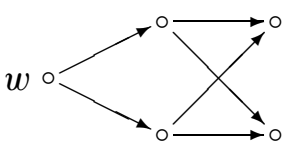

Frame $\mathcal{X}$

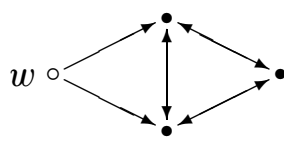

Frame $\mathcal{Y}$

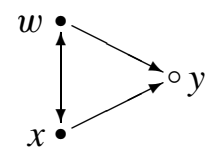

Frame $Z$

Figure 1: Three frames.

The inclusions $\mathcal{F}\left(\mathrm{A}_{\mathbf{4}}^{\Delta}\right) \subset \mathcal{F}\left(\mathrm{A}_{\mathbf{4} b}^{\Delta}\right)$ and $\mathcal{F}\left(\mathrm{A}_{\mathbf{5}}^{\Delta}\right) \subset \mathcal{F}\left(\mathrm{A}_{\mathbf{5} b}^{\Delta}\right)$ are strict, since the frame $\mathcal{X}$ in Figure 1 validates $\left(\mathrm{A}_{\mathbf{5} b}^{\Delta}\right)$ and $\left(\mathrm{A}_{\mathbf{4} b}^{\Delta}\right)$, but falsifies $\left(\mathrm{A}_{\mathbf{4}}^{\Delta}\right)$ and $\left(\mathrm{A}_{\mathbf{5}}^{\Delta}\right)$ at $w$. (Reflexive and irreflexive points are depicted by $\bullet$ and $\circ$, respectively.)

Finally, no other inclusions hold that do not follow from our diagram. To show this, it suffices to refute the inclusions $\mathfrak{E} \mathfrak{u c l} \subseteq \mathcal{F}\left(\mathrm{A}_{4}^{\Delta}\right)$ and $\mathfrak{T} \mathfrak{r a n} \subseteq \mathcal{F}\left(\mathrm{A}_{\boldsymbol{5}_{b}}^{\Delta}\right)$. In Figure 1 , the euclidean frame $\mathcal{Y}$ falsifies $\left(\mathrm{A}_{4}^{\Delta}\right)$ at $w$, whereas the transitive frame $Z$ falsifies $\left(A_{5 b}^{\Delta}\right)$ at $w$.

It is worth noting that if we modify the frame $Z$ by making $w$ irreflexive and $y$ reflexive then we obtain a frame possessing, as Cresswell [3] proved, the following interesting property: its $\square$-logic is not equal to Ver, does not contain $\mathbf{T}$, but necessity is $\Delta$-definable in it by $\square A=[\Delta A \&(A \leftrightarrow \Delta \Delta A)]$.

Theorem 5.3 For $\mathfrak{S} \in\{\mathbf{4}, \mathbf{4} b\}$ (respectively, $\mathfrak{S} \in\left\{\mathbf{5}, \mathbf{5} b, \mathbf{5}^{\prime}\right\}$ ), the axiom $\left(\mathrm{A}_{\mathfrak{S}}^{\Delta}\right)$ expresses the transitivity (respectively, euclideanness) property of reflexive frames. Precisely, a reflexive frame $F$ validates $\left(\mathrm{A}_{\mathfrak{S}}^{\Delta}\right)$ if and only if $F$ is transitive (respectively, euclidean).

Proof: $\quad$ Recall that, in the presence of reflexivity, necessity is $\Delta$-definable by $\square A=$ $A \& \Delta A$. This induces a translation $\operatorname{Tr}$ from $\square$ - into $\Delta$-language which respects Boolean connectives and $\operatorname{Tr}(\square A):=\operatorname{Tr}(A) \& \Delta \operatorname{Tr}(A)$. Clearly, $F \models A \Leftrightarrow F \models$ $\operatorname{Tr}(A)$, for any reflexive frame $F$ and any $\square$-formula $A$. Therefore, in the logic $\mathbf{T}$, $\square$-formulas $A$ and $\operatorname{tr}(\operatorname{Tr}(A))$ are equivalent.

Now we are ready to prove our theorem. For $\mathfrak{S} \in\left\{\mathbf{4} b, \mathbf{5} b, \mathbf{5}^{\prime}\right\}$ the claim follows immediately from completeness of axiomatization of $\mathbf{S} \mathbf{4}^{\Delta}$ and $\mathbf{S 5}^{\Delta}$ proved in 8 and [9]. Consider, for instance, the case $\mathfrak{S}=\mathbf{4} b$.

Take an arbitrary reflexive frame $F$. If $F$ is transitive then $F \models \mathbf{S 4}$, so $F \models \mathbf{S 4}^{\Delta}$ and $F \models\left(\mathrm{A}_{\mathbf{4}}^{\Delta}\right)$. To prove the converse, assume that $F \models\left(\mathrm{A}_{\mathbf{4 b}}^{\Delta}\right)$, then $F \models \mathbf{S 4}^{\Delta}$. Due to completeness of $\mathbf{S 4}^{\Delta}$, from $\mathbf{S} \mathbf{4} \vdash \operatorname{tr}\left(\operatorname{Tr}\left(\mathrm{A}_{\mathbf{4}}^{\square}\right)\right)$ it follows that $\mathbf{S 4}^{\Delta} \vdash \operatorname{Tr}\left(\mathrm{A}_{\mathbf{4}}^{\square}\right)$, so $F \models$ $\operatorname{Tr}\left(\mathrm{A}_{4}^{\square}\right)$ and $F \models\left(\mathrm{A}_{4}^{\square}\right)$, thus $F$ is transitive.

The claim for $\mathfrak{S}=\mathbf{5}$ follows from equivalence of $\left(A_{5}^{\Delta}\right)$ and $\left(A_{\mathbf{5}^{\prime}}^{\Delta}\right)$.

For $\mathfrak{S}=\mathbf{4}$ the argument is: on the one hand, $\left(A_{4}^{\Delta}\right)$ is stronger than $\left(A_{\mathbf{4}}^{\Delta}\right)$, by Corollary 5.2. on the other, it is not too strong to go beyond $\mathbf{S 4}^{\Delta}$, that is, $\left(A_{\mathbf{4}}^{\Delta}\right) \in$ $\mathbf{K 4}^{\Delta} \subset \mathbf{S} \mathbf{4}^{\Delta}$. Hence, $\left(\mathrm{A}_{\mathbf{4}}^{\Delta}\right)$ can be treated like $\left(\mathrm{A}_{\mathbf{4} b}^{\Delta}\right)$ above.

We conclude with a remark on axiomatic systems presented in Section 2. According to Corollary 5.2. the axiom $\left(A_{4}^{\Delta}\right)$ is stronger than $\left(A_{\mathbf{4} b}^{\Delta}\right)$. Hence $\left(A_{\mathbf{4} b}^{\Delta}\right)$ can be replaced by $\left(A_{4}^{\Delta}\right)$ in the axiomatization of $\mathbf{S 4}^{\Delta}$. Humberstone 4 conjectured that $\mathbf{K 4}^{\Delta}$ can be axiomatized using the simpler axiom $\left(A_{4 b}^{\Delta}\right)$ instead of $\left(A_{4}^{\Delta}\right)$. However, Kuhn [6] 
refuted the conjecture by providing the frame $X$ (see Figure 1 above) which separates these axioms. For the same reason, in the axiomatization of $\mathbf{K 5}^{\Delta}$, the axiom $\left(A_{5}^{\Delta}\right)$ cannot be replaced by $\left(A_{5 b}^{\Delta}\right)$ (for the frame $\mathcal{X}$ separates these axioms too). At the same time, we can replace $\left(A_{\mathbf{5}^{\prime}}^{\Delta}\right)$ by $\left(A_{5}^{\Delta}\right)$ in the axiomatization of $\mathbf{S 5}^{\Delta}$, since $\mathbf{K 5}^{\Delta} \vdash\left(A_{\mathbf{5}^{\prime}}^{\Delta}\right)$ by Corollary 5.2 and Theorem 2.2. Here is an open question: Can we replace $\left(A_{5}^{\Delta}\right)$ by $\left(A_{\mathbf{5}^{\prime}}^{\Delta}\right)$ in the axiomatization of $\mathbf{K 5}^{\Delta}$ ? 
Acknowledgments The research is partially supported by the Russian Foundation for Basic Research. I would like to thank Max Cresswell for bringing some publications on noncontingency logic to my attention. I am grateful to S. N. Artemov for help during the reseach and to V. B. Shehtman for fruitful discussions on the subject. I also thank the referee for detailed comments on the manuscript and useful suggestions.

\section{REFERENCES}

[1] Boolos, G., Logic of Provability, Cambridge University Press, Cambridge, 1993. Zbl 0891.03004|MR 95c:03038 4

[2] Chagrov, A., and M. Zakharyaschev, Modal Logic, Oxford Science Publications, Oxford, 1997.Zbl 0871.03007|MR 98e:03021 2.13

[3] Cresswell, M. J., "Necessity and contingency," Studia Logica, vol. 47 (1988), pp. 45149. Zbl 0666.03015 MR 90d:03032 1,5

[4] Humberstone, I. L., "The logic of non-contingency," Notre Dame Journal of Formal Logic, vol. 36 (1995), pp. 214-29. Zbl 0833.03004 MR 96g:03029 1, 2,12,3,3, 3, 3. $4,4,4,15,5$

[5] Kuhn, S. T., "Minimal non-contingency logic," Notre Dame Journal of Formal Logic, vol. 36 (1995), pp. 230-34.Zbl 0833.03005|MR 96g:03030 1, 1, 2,,2,2

[6] Kuhn, S. T., abstract in "Mathematical Reviews," MR 969:03029. 5

[7] Makinson, D., "Some embedding theorems for modal logic," Notre Dame Journal of Formal Logic, vol. 12 (1971), pp. 252-54.Zbl 0211.01203|MR 52:2824 3

[8] Montgomery, H., and R. Routley, "Contingency and non-contingency bases for normal modal logics," Logique et Analyse, vol. 9 (1966), pp. 318-28. Zbl 0294.02008|MR 36:6270 1.2.12.15

[9] Montgomery, H., and R. Routley, "Non-contingency axioms for $\mathbf{S 4}$ and S5," Logique et Analyse, vol. 11 (1968), pp. 422-24. Zbl 0169.30003 MR 38:4288 1. 2.|2.5

[10] Montgomery, H., and R. Routley, "Modalities in a sequence of normal non-contingency modal systems," Logique et Analyse, vol. 12 (1969), pp. 225-27.

Zbl 0196.00902||MR 43:7305 1

Department of Mathematical Logic

Faculty of Mathematics and Mechanics

Moscow State University

119899 Moscow

RUSSIA

email: zolin@lpcs.math.msu.ru 